Original Research Paper

\title{
A Framework for Real-Time Healthcare System Performance in Developing Countries
}

\author{
${ }^{1}$ Razi Ahmed, ${ }^{1 *}$ Eiad Yafi, ${ }^{2}$ Mazliham Mohd Su'ud, ${ }^{3}$ Muhammad Mansoor Alam and ${ }^{1}$ Muhammad Faizan \\ ${ }^{1}$ Universiti Kuala Lumpur, Malaysian Institute of Information Technology, Kuala Lumpur, Malaysia \\ ${ }^{2}$ Universiti Kuala Lumpur, Malaysia France Institute, Kuala Lumpur, Malaysia \\ ${ }^{3}$ Institute of Business Management Karachi, Pakistan
}

\section{Article history}

Received: 02-05-2020

Revised: 07-09-2020

Accepted: 23-09-2020

Corresponding Authors: Eiad Yafi

Malaysian Institute of Information Technology, Universiti Kuala Lumpur, Kuala Lumpur, Malaysia Email: eiad@unikl.edu.my

\begin{abstract}
This study proposes a conceptual structure for the success of the real-time healthcare program in developing countries. Whilst developing and some developed countries face a significant challenge in adopting the application of real-time healthcare, this work aims to analyze the perspectives of healthcare practitioners in Malaysia. Since 1997, Malaysia has been putting efforts to introduce a real-time healthcare system, but due to geographical constraints and shortage of physicians, people in the rural area often have a hard time accessing quality healthcare services. This research proposes a remote patient's management framework to predict user's behaviour by three outputs of the real-time healthcare system performance: Net gain, system expectation and acceptance. Remote patient management system offering access to patient's data regardless of the location of patients and physicians is the only way forward for healthcare providers in developed and developing countries facing a shortage of healthcare services due to geographical constraints natural disasters of pandemics. We emphasize that there should be no compromise when it comes to the privacy of the patient's data in the proposed system. However, recent technologies such as the Internet of Things and Blockchain is deployed to ensure utmost privacy and security of patient's data.
\end{abstract}

Keywords: Real-Time Healthcare, System Performance, User Interaction, Developing Countries

\section{Introduction}

Medical technology faces significant challenges as the healthcare services have been improved globally due to the innovation of the real-time healthcare application. The real-time healthcare application is rapidly being used by the healthcare practitioner as an alternative method in healthcare services and it provides a lot of benefits and advantages in improving patient's healthcare at a distance.

Real-time healthcare is the delivery of healthcare services and application based on Information and Communication Technologies (ICT) tools to improve people's healthcare in their daily life (Almathami et al., 2020). Real-time healthcare is defined broadly (Field, 1996) as "the use of electronic information and communication technologies to provide and support healthcare when distance separates the participants". The use of real-time healthcare has been growing globally, especially in developed countries and it is increasingly adopted in developing countries like Malaysia. This paper outlines a research study that examines the user's real-time healthcare system performance and adoption with the development of the user interaction framework model. In this study, only the healthcare practitioner will be assessed as the principal user of real-time healthcare applications. The healthcare practitioner used the realtime healthcare application to evaluate the patient in healthcare services by making a medical decision, remote sensing and monitoring in the real-time and store and forward Management of the patient by a distance. The advancement and modernization of real-time healthcare need to be matched up with innovation adoption and technology acceptance within the key user especially healthcare practitioner for its effectiveness and optimization in usages (Ahmadi et al., 2015; Hak et al., 2020; Jackson and McClean, 2012). In experimental assessment (Saha et al., 2017), a real-lifetime series Telehealth data obtained from patients with chronic heart disease is used. The experimental results indicate that the 
proposed program provides a perfect recommendation accuracy and offers an efficient way of minimizing the likelihood of inaccurate recommendations as well as reducing the workload for patients with heart disease in carrying out routine body checks.

\section{A. Real-Time Healthcare in Developing Countries}

Developing countries are still in the early stages of real-time healthcare implementation and only a few countries have begun their initial project to use real-time healthcare applications as part of technology updated in healthcare services. In contrast, real-time healthcare applications have been widely used in developed countries. The implementation of real-time healthcare is recognized to provide a lot of benefits and advantages as it is determining the enhancement to provide a better quality of healthcare under continuous pressure to reduce cost, especially in developing countries (Mackert et al., 2014).

Real-time healthcare for reforming the healthcare services industry can take many forms. In this study, the real-time healthcare application has been identified as improving healthcare access to the people in a remote location, thus will generate a significant impact on service providing and economies in developing countries (Frost and Sullivan, 2014). It is reported that in the Asia Pacific region alone, real-time healthcare equipment has generated sales of US 773 million and it was increased every year. The developing countries experience an improvement of real-time healthcare implementation, which is driven by a factor of inadequate facilities and infrastructure, ratios between the healthcare practitioner and patients, ageing population, high spread of viruses and chronic diseases (Hak et al., 2020). The success of implementing the realtime healthcare services in developing countries consists of how the local people's support expertise in the technology, proper maintenance and management of the system, good ICT infrastructure and a security framework for the users (Lalrochunga et al., 2020). This is a new, universal and extensible cloud platform system for tracking, diagnosing and predicting aspects of individuals' health using, for example, wearable medical devices toward the goal of personal health management, particularly for the elderly (Liu et al., 2019).

\section{B. Real-Time Healthcare in Malaysia}

Malaysia established the Multimedia Super Corridor (MSC) in 1996 to enhance the use of ICT in the country. There were a total of 7 flagships to drive Malaysia into the ICT limelight and real-time healthcare was chosen to be one of them (Judi et al., 2010). Ministry Of Health (MOH) (Yew et al., 2020) Malaysia, a constitution body under Malaysia's government, was responsible as a healthcare services provider for citizens and initiated national real-time healthcare projects by publishing official Real-time Healthcare Blueprints in 1997. Real-time healthcare Blueprint is an informatics framework model for the beginning implementation of real-time healthcare projects in Malaysia. It emphasized the healthcare revolutionary from the traditional system to advanced system by harnessing the capability of ICT potential, which is promoting greater access to the rural and remote areas for healthcare delivery (Ministry Of Health (MOH) Malaysia) (Yew et al., 2020). An Internet of Things (IoT)-based remote patient monitoring network that is capable of maintaining the validity of an Electrocardiogram (ECG) in real-time. Message Queuing Telemetry Transport (MQTT) protocol is used to forward the ECG from the proposed device to the webserver in real-time (Yew et al., 2020). The PubMed, IEEEXplore and Science Direct electronic databases were listed as the principal sources of data. Specific online repositories were also investigated, such as ACM Digital Library, Cochrane or the Science Social Citations Citation Index (SSCI) Online. Still, they returned zero (ACM) and one result (Cochrane, SSCI) respectively (Katrakazas et al., 2020).

The development of real-time healthcare in Malaysia is in line with the rapid advancement of real-time healthcare services; however, (Judi et al., 2010), the hospital in Malaysia still in the early stages of the implementation and adoption. Since the early 2000s, Malaysian Ministry of Health has deployed a real-time healthcare application to be used for healthcare delivery that consists of Telehealth ICT project, Teleconsultation, Teleprimary care, Teleradiology and Integration of the Healthcare Management System. However, there is an argument that the real-time healthcare required proper Management in the project implementation due to any issues of the technology acceptance and adoption among the users, cost-effectiveness and integration of the projects (Kam et al., 2020).

\section{Research Approach}

The research approach applied in this study evaluates real-time healthcare system performance by surveying healthcare practitioners based on the user interaction model. User interaction is a subset to Human-Computer Interaction (HCI) that explains how users act with the computerized system and how the automated system act to users (Ahmed et al., 2018), which is vice versa in performing the tasks related to technology (Shneiderman and Plaisant, 2010). In HCI, persons or a group need to be satisfied in the most effective way when they do the jobs or working with the computerized system to get the optimized performance results. 


\section{A. Human-Computer Interaction (HCI)}

The goals of Human-Computer Interaction (HCI) study are to make systems more usable, more useful and to provide users with experience fitting, which is related to the current technology phenomenon (Alves et al., 2020). According to (Shneiderman and Plaisant, 2010; Wali et al., 2020), HCI can be evaluated by five key pillars; system response time, the time required by users to learn the system, ability of users to remember what they learned, error rate and subjective user satisfaction to use the system. In healthcare services, HCI studies can facilitate a goal of design revision and improve the user's performance (healthcare practitioner) usage. Apart from that, HCI also provides a valuable method to improvise system implementation and performance in the future (Wali et al., 2020).

\section{B. A Proposed Framework for Real-time Healthcare System Performance (TSP)}

The proposed framework depicts the real-time healthcare application system performance based on the view of healthcare practitioner by applying an integrated factor pertaining on the user satisfaction and user technology acceptance using a hybrid model from Web-Based Information System Success Model (WISSM) by (Garrity, 2005) and The Unified Theory of Acceptance and Use of Technology Method (UTAUT) (Tao et al., 2020; Sutton et al., 2020).

In this research, the user satisfaction factors comprise the characteristics of satisfaction to use the real-time healthcare application based on the interface and process that will impact on performance measures in using the technology. According to (Chau and Hu, 2002), the user satisfaction context concentrates on essential aspects of the technology under investigation that will provide a reference point for healthcare practitioners to use healthcare technology. Furthermore, the user technology acceptance refers to the technology implementation in a specific healthcare practitioner environment take place and also underlying to organizational performance setting that affected to decision making in corporate Management.

\section{Web-Based Information System Success Model (WISSM)}

Web-Based Information Systems Success Model (WISSM) is a model framework in an information system that was updated from the extension of Delone and Mclean's information systems success model, Technology Acceptance Model (TAM) and the Garrity and Sander's Model of IS Success as underpinning theoretical framework (Sutton et al., 2020; Wixom and Todd, 2005). The WISSM success was developed to examine a consumer purchasing decision with a focus on
User Satisfaction that consists of three fundamental components; Task Support Satisfaction (TSS), Decision Support Satisfaction (DSS) and Interface Satisfaction.

The approach taken by (Garrity, 2005) in making a WISSM success is goal-oriented methods; end-users need to be supported by a high-quality performance system to perform their tasks or applications in using the Information System (IS) technology and it should be related to the end-users job function which is varied (Sutton et al., 2020).

Figure 1 shows the enhanced WISSM originated from (Garrity, 2005). The efficiency of the application in the information system interface is measured using the Interface Satisfaction, which is direct to DSS and TSS to accomplish the goals of interface design to support the users in using the system. The effectiveness of the methods is measured through the DSS, which is direct to the TSS. The overall efficiency and effectiveness of the WISSM are measured via the TSS, which is direct to the behavioural intention to use the Electronic Commerce (EC) website.

\section{The Unified Theory of Acceptance and Use of Technology Method (UTAUT)}

Healthcare technology research is increasingly becoming a crucial theoretical instrument for the researchers investigating technology acceptance which helps members who are actively involved in IT projects such as administrators, designers, purchasers, vendors and others wit to get a broader understanding of successful criterion in implementation and project execution (Holden and Karsh, 2010). UTAUT, which manipulates the TAM variables, allows the objectives of purpose to access the vital role of encouraging technology acceptance for the use of creativity and invention to maximize the IT usage by the person or company. The success of the new change is dependent on technology acceptance by the right consumer adoption. Tao et al. (2020) has developed an integrated model to identify and validate user acceptance towards new technology, which is known as the Unified Theory of Acceptance and Use of Technology (UTAUT). There were eight models consist of the theory of reasoned action, technology acceptance model, the motivational model, the method of planned behaviour, a model combining the technology acceptance model and theory of planned behaviour, the model of personal computer utilization into one inclusive UTAUT model design to identify individual acceptance related to the adoption of new technology.

According to the eight variables (Hawash et al., 2020) in UTAUT, it helps to determine the technology acceptance and use and it enables a factor in understanding future technology adoption. Figure 2 shows the original UTAUT model (Venkatesh et al., 2011). 


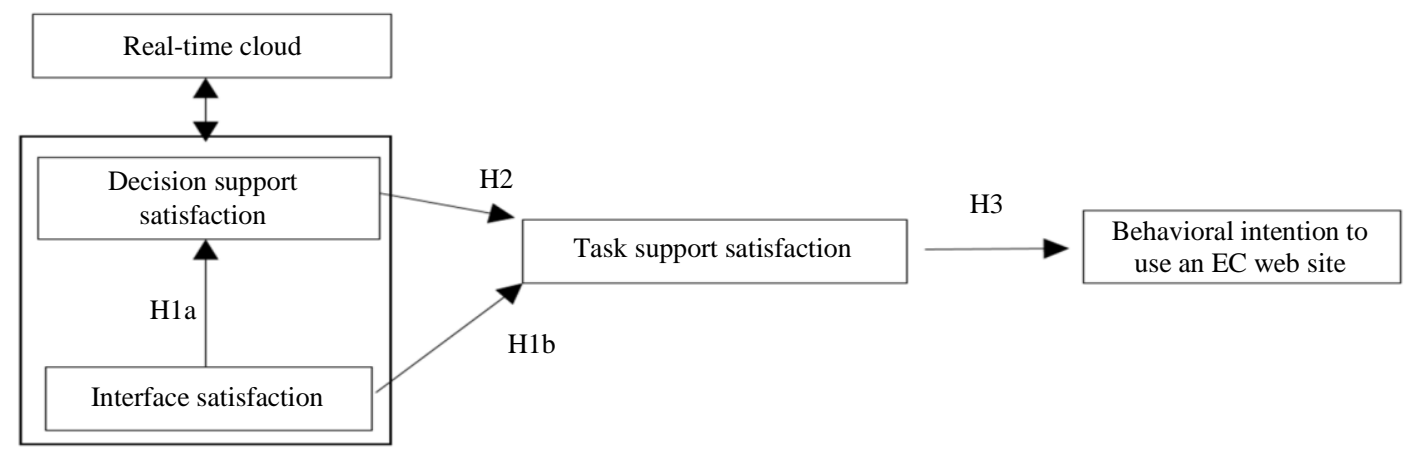

Fig. 1: The Web-based Information Systems Success Model (WISSM)

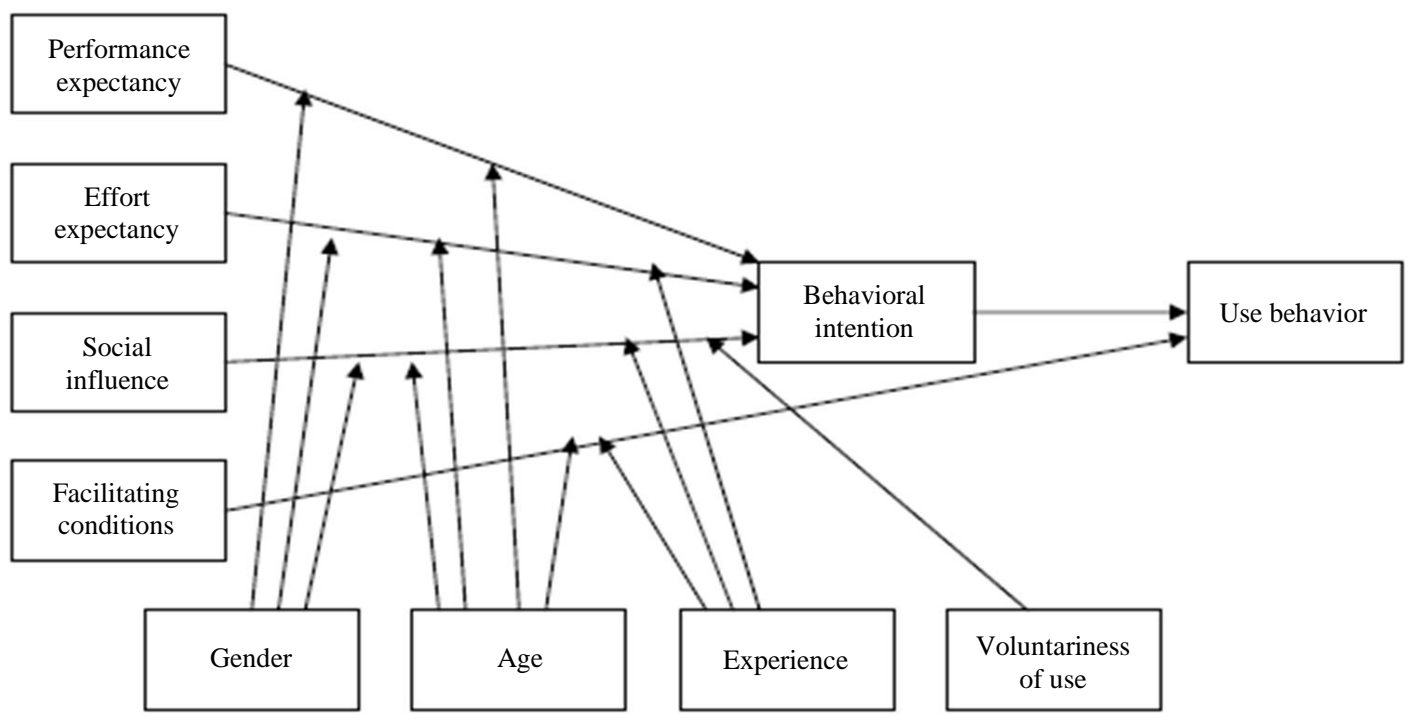

Fig. 2: The original UTAUT model

Based on (Tao et al., 2020), four key constructs exist to be used to perform in identifying the user acceptance of new technology by consumers. The four key construct consists of performance expectancy, effort expectancy, social influence and facilitating conditions. The UTAUT model established a beneficial mechanism for managers and administrations of the organization to evaluate the favourable outcome of the innovation, new IT introduction and new technology use. For this research, the revised UTAUT by (Venkatesh et al., 2011), which was used to study Electronic Medical Record (EMR) system, will be applied to test for real-Time healthcare Systems Performance (TSP) that currently being used in Malaysia. The moderator consists of gender, age, experience and voluntariness of use from the original UTAUT need to be modified and significant with the real-time healthcare application context.

\section{E. The Conceptual Framework}

Three factors from WISSM consisting of Interface Satisfaction, Decision Support Satisfaction and Task
Satisfaction are integrated with three variable factors from UTAUT, which include performance expectancy, effort expectancy and facilitating conditions (Amadi-Obi et al., 2014). Figure 3 shows the proposed conceptual framework to measure the realtime healthcare system performance. Besides, the security and privacy factor (Falter and Hadwich, 2020; Díaz-Martín et al., 2020) have been added in the framework as part of performance measures in real-time healthcare system performance. The output of real-time healthcare system performance utilizing three measurement areas of real-time healthcare net benefit, real-time healthcare system expectation and real-time healthcare adoption (Musa Jaber et al., 2014).

\section{F. Research Design}

In this study, the assessment of real-time healthcare system performance is carried out in hospitals at Kelang Valley Malaysia consists of 2 states: Wilayah Persekutuan (Kuala Lumpur and Putrajaya) and Selangor. In Malaysia, there are few studies related to 
real-time healthcare and most of the surveys in healthcare services focus on the adoption of Hospital Information System (HIS) (Lee et al., 2012) and Electronic Medical Record (EMR) (Marzuki et al., 2012). The past research by (Judi et al., 2010) only evaluate a few hospitals in Kelang Valley for the realtime healthcare adoption, which is not cover all the MOH's public hospital in the region and it was only based on the professional individual's motivating factors to use the real-time healthcare technology.

Esmaeilzadeh and Sambasivan (2012) claimed that there are evidence and justification that healthcare practitioners are unwilling to use the Clinical IT (new medical technologies) in their daily works because it has changed the traditional practice pattern in medical services. They also argued that the factors affecting adoption and acceptance in using the healthcare technologies within healthcare practitioners are incorporating the characteristics of a physician, IT knowledge level and the features of technologies/systems (Saha et al., 2017).

Based on the report by (Rossman et al., 2020; Harris et al., 2020), the most commonly real-time healthcare applications used globally in the healthcare services consist of teleradiology, teledermatology, telepathology and telepsychiatry. In this research, the assessment is focused on the broad range of real-time healthcare applications comprised of tele-monitoring, tele-diagnosis, tele-consultation, tele-radiology, teledermatology tele-pathology, educational, electronic medical record and others (will be specified by the healthcare practitioner under investigation during the primary fieldwork) (Ahmed et al., 2019).

In this research study, a context-based approach is applied to observe the real-time healthcare application used in Malaysia, utilizing a self-administrated questionnaire to examine the healthcare practitioners using the technology. The questionnaire content design is based on the pertinent theories proposed and verified by (Saha et al., 2017). The questionnaire survey was distributed to the healthcare practitioners who have used the real-time healthcare application in Malaysia in 2016 and before. According to (Creswell, 2014), by using the quantitative research approach on the selfadministrated questionnaire, the relationship among the integrating factors can be determined based on the assumption of deductive theories and then generalize the findings (Saher et al., 2014).

\section{G. Pilot Test}

Data collection of this study was intended to be carried out quantitatively by conducting semi-structured survey questionnaires during the first quarter of 2016 but was postponed for funding constraints. Before the data collection, a pilot study was conducted in the third quarter of 2015 using 15 survey questionnaires. The survey respondents for the pilot study included 12 medical officers and three senior nurses. These pilot studies were used to test the validity and general understanding of the questionnaire's item. The Cronbach Alpha test using SPSS version 20 is used to measure the reliability of the items factor measures in the framework. Table 1 shows the results of the Cronbach Alpha for the pilot study. The values of the Cronbach Alpha's are ranging from 0.716 to 0.976 . All the item factors demonstrated excellent reliability results above the suggested value of 0.7 (Pallant, 2010). The results from the pilot study will be used to improve the questionnaire design and to understand the real-time healthcare system performance among healthcare practitioners in Developing Countries.

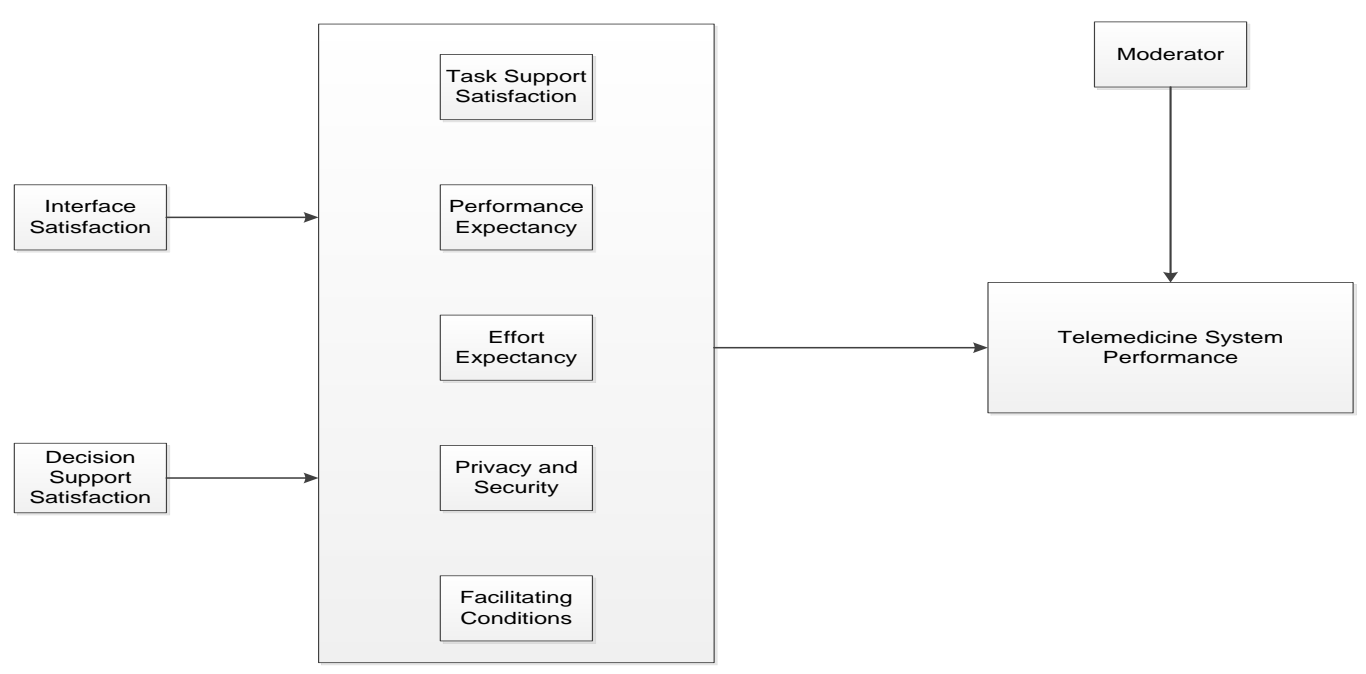

Fig. 3: The conceptual framework of real-time healthcare system performance in developing countries 
Table 1: Cronbach alpha results for a pilot study

\begin{tabular}{ll}
\hline Item factor & Cronbach alpha \\
\hline Real-time healthcare net benefit & 0.922 \\
Real-time healthcare system expectation & 0.965 \\
Real-time healthcare Adoption & 0.952 \\
Interface satisfaction & 0.976 \\
Decision support satisfaction & 0.976 \\
Task support satisfaction & 0.935 \\
Performance expectancy & 0.971 \\
Effort expectancy & 0.969 \\
Facilitating conditions & 0.716
\end{tabular}

\section{Discussion}

A research gap was highlighted by (Díaz-Martín et al., 2020) in assessing the performance measure on the user interaction and design system flow in the healthcare context. According to (Wixom and Todd, 2005), there are only a few studies related to technology performance by integrating user satisfaction and technology acceptance in the perception of Information System (IS) success. The integration of user satisfaction and technology acceptance builds a strong relationship that distinguishes belief and attitude from the user perspective to improve their performance in using the new technology. The integration of user satisfaction and technology acceptance can help to build a bridge from design and implementation with the system characteristics; therefore, it can be used as a streams tool for performance measurement of real-time healthcare applications in healthcare contexts. Thus, the outcome of this research study provides insightful knowledge to a researcher in academia and system implementers of realtime healthcare projects, service providers and healthcare administrator are in developing countries. The framework proposed in this study predicts user behaviour by three outputs of the real-time healthcare system performance. AI is used to discover new connections between genetic codes and push surgeryassisted robots, artificial intelligence reinvents and revitalizes modern healthcare through machines that can predict, understand, learn and act. Our proposed framework is utilizing artificial intelligence to predict the user's behaviour in the e-health system. The results obtained from the pilot test depicts that the framework has a positive impact on health systems processes.

\section{Conclusion and Future Work}

It is significant to understand the real-time healthcare system performance to know their impact on healthcare services in Malaysia. This research is aimed to develop a research framework that understands the effects of user interaction that will be affected by real-time healthcare system performance by utilizing three output measurements: Net benefit, system expectation and adoption. This research is still in the early stages.
However, the second phase of data collection has started right before Covid19 lock-down. We aim to learn from the future data analysis for the validation of item factors and redraw a relationship for the proposed framework. The built-in real-time patient monitoring system has worked on both private and public networks. The main contributions of this research are the expert system that provided the adoption, benefit, system expectation. The recommended framework can be applied in the hospital. The integration of AI in healthcare is changing the way of automating treatments and provides tremendous improvements. However, the contribution of our paper is that it is taking e-healthcare to new heights by performing real-time analytics on the available data to enable the caregivers improving customized treatment programs, monitoring treatment and improve the overall health of patients. Health systems shift from an incentive model focused on fees, admissions and revenues to concentrate on reliable delivery of evidence-based treatment at the right time, the right place, the right use of the right human environment.

It is possible to integrate real-time machine learning and $\mathrm{AI}$ algorithms into the proposed framework as future work so that the process can run more efficiently. This framework can also benefit from the novel could service to collect real-time feedback assessment data on different health parameters.

\section{Acknowledgement}

Foremost, I would like to express my sincere gratitude to my advisor Prof. Eiad Yafi for the continuous support of my PhD study and research, for his patience, motivation, enthusiasm and immense knowledge. His guidance helped me in all the time of research and writing of this article.

\section{Author's Contributions}

Razi Ahmed: Conceived the ideas of experimental design of the study, performed experiments/data collection, data analysis and interpretation and writingoriginal draft preparation.

Eiad Yafi: Conceived the ideas of experimental design of the study, writing-review and editing and supervision.

Mazliham Mohd Su'ud: Data analysis and interpretation and supervision.

Muhammad Mansoor Alam: Writing-review and editing and supervision.

Muhammad Faizan: Performed experiments/data collection.

\section{Conflict Statement}

The Authors declares that there is no conflict of interest. 


\section{References}

Ahmadi, H., Ibrahim, O., \& Nilashi, M. (2015). Investigating a new framework for hospital information system adoption: a case on Malaysia. Journal of Soft Computing and Decision Support Systems, 2(2), 26-33.

Ahmed, R., Faizan, M., \& Burney, A. I. (2019, December). Process Mining in Data Science: A Literature Review. In 2019 13th International Conference on Mathematics, Actuarial Science, Computer Science and Statistics (MACS) (pp. 1-9). IEEE.

Ahmed, R., Hussain, M., Rahmani, T. S., Mansoor, A., \& Ali, M. L. (2018). Minimization of security issues in cloud computing. Journal of Information Communication Technologies and Robotic Applications, 3-40.

Almathami, H. K. Y., Win, K. T., \& Vlahu-Gjorgievska, E. (2020). Barriers and facilitators that influence telemedicine-based, real-time, online consultation at patients' homes: systematic literature review. Journal of medical Internet research, 22(2), e16407.

Alves, T., Natálio, J., Henriques-Calado, J., \& Gama, S. (2020). Incorporating personality in user interface design: A review. Personality and Individual Differences, 155, 109709.

Amadi-Obi, A., Gilligan, P., Owens, N., \& O’Donnell, C. (2014). Telemedicine in pre-hospital care: a review of telemedicine applications in the prehospital environment. International journal of emergency medicine, 7(1), 29.

Chau, P. Y., \& Hu, P. J. H. (2002). Investigating healthcare professionals' decisions to accept telemedicine technology: an empirical test of competing theories. Information \& management, 39(4), 297-311.

Creswell, J. W. (2014). A concise introduction to mixed methods research. SAGE publications.

Díaz-Martín, A. M., Schmitz, A., \& Yagüe Guillén, M. J. (2020). Are health e-mavens the new patient influencers?. Frontiers in Psychology, 11, 779.

Esmaeilzadeh, P., \& Sambasivan, M. (2012). Healthcare professionals' adoption of clinical IT in hospital: a view of relationship between healthcare professionals and hospital. Management, 2(5), 161-170.

Falter, M., \& Hadwich, K. (2020). Customer service well-being: scale development and validation. The Service Industries Journal, 40(1-2), 181-202.

Field, M. J. (Ed.). (1996). Telemedicine: A guide to assessing telecommunications for health care. National Academies Press.

Frost \& Sullivan (2014), Growth and innovation in the healthcare industry: Trends, opportunities and challenges.

http://ww2.frost.com/files/2214/2200/8908/UnitedHeal th_HC_Trends_Discussion.pdf
Garrity, A. (2005). Validation of publication of new names and new combinations previously effectively published outside the IJSEM. Int $\mathrm{J}$ Syst Evol Microbiol, 55, 2235-2238.

Hak, F., Oliveira, D., Abreu, N., Leuschner, P., Abelha, A., \& Santos, M. (2020). An OpenEHR Adoption in a Portuguese Healthcare Facility. Procedia Computer Science, 170, 1047-1052.

Harris, C., Carson, G., Baillie, J. K., Horby, P., \& Nair, H. (2020). An evidence-based framework for priority clinical research questions for COVID19. Journal of global health, 10(1).

Hawash, B., Asma'Mokhtar, U., Yusof, Z. M., \& Mukred, M. (2020). The adoption of electronic records management system (ERMS) in the Yemeni oil and gas sector. Records Management Journal.

Holden, R. J., \& Karsh, B. T. (2010). The technology acceptance model: its past and its future in health care. Journal of biomedical informatics, 43(1), 159-172.

Jackson, D. E., \& McClean, S. I. (2012). Trends in telemedicine assessment indicate neglect of key criteria for predicting success. Journal of health organization and management.

Judi, H. M., Sahari, N., Mohamed, H., \& Razak, A. A. (2010). The motivating factors for telemedicine application in Klang Valley. Asia-Pacific Journal of Information Technology and Multimedia, 8(1).

Kam, K. L., Yew, H. T., \& Hau, Y. W. (2020). Introduction of Telecardiology and Challenges in Developing Countries. In Cardiovascular Engineering (pp. 125-142). Springer, Singapore.

Katrakazas, P., Pastiadis, K., Bibas, A., \& Koutsouris, D. (2020). A General Systems Theory Approach in Public Hearing Health: Lessons Learned From a Systematic Review of General Systems Theory in Healthcare. IEEE Access, 8, 53018-53033.

Lalrochunga, D., Parida, A., \& Choudhury, S. (2020). Sustainability of UAVs in developing countries: Prospects and challenges. Journal of Discrete Mathematical Sciences and Cryptography, 23(1), 237-248.

Lee, H. W., Ramayah, T., \& Zakaria, N. (2012). External factors in hospital information system (HIS) adoption model: A case on Malaysia. Journal of Medical Systems, 36(4), pp. 2129-2140.

Liu, Y., Zhang, L., Yang, Y., Zhou, L., Ren, L., Wang, F.,... \& Deen, M. J. (2019). A novel cloud-based framework for the elderly healthcare services using digital twin. IEEE Access, 7, 49088-49101.

Mackert, M., Champlin, S. E., Holton, A., Muñoz, I. I., \& Damásio, M. J. (2014). eHealth and health literacy: a research methodology review. Journal of Computer-Mediated Communication, 19(3), 516-528. 
Marzuki, N. M., Ismail, S., Mohsein, N. A. S. A., \& Ehsan, F. Z. (2012, July). Evaluation of Telehealth implementation in government primary health clinics-A study protocol. In 2012 International Conference on Green and Ubiquitous Technology (pp. 144-148). IEEE.

Musa Jaber, M., Abd Ghani, M. K., \& Suryana Herman, N. (2014). A REVIEW OF ADOPTION OF TELEMEDICINE IN MIDDLE EAST COUNTRIES: TOWARD BUILDING IRAQI TELEMEDICINE FRAMEWORK. Science International, 26(5).

Pallant, J. (2010). SPSS Survival Manual. 4th edition. McGraw Hill Education, London.

Rossman, H., Keshet, A., Shilo, S., Gavrieli, A., Bauman, T., Cohen, O.,.. \& Segal, E. (2020). A framework for identifying regional outbreak and spread of COVID-19 from one-minute populationwide surveys. Nature Medicine, 26(5), 634-638.

Saha, B., Gupta, S., Phung, D., \& Venkatesh, S. (2017). A framework for mixed-type multioutcome prediction with applications in healthcare. IEEE journal of biomedical and health informatics, 21(4), 1182-1191.

Saher, S., Ahmed, R., Faizan, M., \& Burney, A. I. (2014). Critical Analysis Relation between Indo-Pak: Kartarpur Corridor. http://www.ijrar.org/viewfull.php?\&p_id=IJRAR2001 131

Shneiderman, B., \& Plaisant, C. (2010). Designing the user interface: Strategies for effective human computer interaction. 5 ed. Pearson Higher Education, Boston.
Sutton, R. T., Pincock, D., Baumgart, D. C., Sadowski, D. C., Fedorak, R. N., \& Kroeker, K. I. (2020). An overview of clinical decision support systems: benefits, risks and strategies for success. NPJ Digital Medicine, 3(1), 1-10.

Tao, D., Wang, T., Wang, T., Zhang, T., Zhang, X., \& Qu, X. (2020). A systematic review and metaanalysis of user acceptance of consumer-oriented health information technologies. Computers in Human Behavior, 104, 106147.

Venkatesh, V., Sykes, T. A., \& Zhang, X. (2011, January). 'Just what the doctor ordered': A revised UTAUT for EMR system adoption and use by doctors. In 2011 44th Hawaii International Conference on System Sciences (pp. 1-10). IEEE.

Wali, R. M., Alqahtani, R. M., Alharazi, S. K., Bukhari, S. A., \& Quqandi, S. M. (2020). Patient satisfaction with the implementation of electronic medical Records in the Western Region, Saudi Arabia, 2018. BMC Family Practice, 21(1), 1-6.

Wixom, B. H., \& Todd, P. A. (2005). A theoretical integration of user satisfaction and technology acceptance. Information systems research, 16(1), 85-102.

Yew, H. T., Ng, M. F., Ping, S. Z., Chung, S. K., Chekima, A., \& Dargham, J. A. (2020, February). IoT Based Real-Time Remote Patient Monitoring System. In 2020 16th IEEE International Colloquium on Signal Processing \& Its Applications (CSPA) (pp. 176-179). IEEE. 